\title{
Arbeitnehmerbeteiligung in mittelständischen Unternehmen
}

\author{
Nadine Schlömer, Rosemarie Kay \\ Wolfgang Rudolph, Wolfram Wassermann
}

Betriebsräte, die gemäß Betriebsverfassungsgesetz gewählt worden sind, bleiben in mittelständischen Betrieben eine Ausnahme. Nach einer Sonderauswertung des IAB-Betriebspanels verfügten im Jahr 2004 nur etwa $10 \%$ der betriebsratsfähigen kleinen und mittleren Betriebe über einen Betriebsrat. Es wäre jedoch ein Fehlschluss, aus diesen Daten zu folgern, dass Beschäftigte in mittelständischen Betrieben keinerlei Möglichkeiten hätten, ihre Interessen zu artikulieren und zu vertreten. Verbreitung und Zuschnitt unterschiedlicher, auch alternativer Formen kollektiver und individueller Interessenvertretung sind das Thema dieses Beitrages.

\section{Einleitung}

Nach dem Betriebsverfassungsgesetz kann in Betrieben mit mindestens fünf abhängig Beschäftigten ein Betriebsrat von den Beschäftigten gewählt werden ( $\$ 1$ BetrVG). Damit ist Mitbestimmung über Betriebsräte in Deutschland bereits in kleinen mittelständischen Betrieben möglich. Anders sieht dies in den meisten anderen europäischen Ländern aus, wo Betriebsräte erst in Betrieben ab 20, 30 oder 50 Arbeitnehmern gewählt werden dürfen (Niedenhoff 2005, S. 9). Um die Bedeutung der Mitbestimmung im deutschen Mittelstand beurteilen zu können, muss neben diesen gesetzlichen Bestimmungen (de jure-Zustand) jedoch auch die tatsächliche Umsetzung der rechtlichen Möglichkeiten (de facto-Zustand) in den Unternehmen betrachtet werden. Tatsächlich zeigt sich, dass nur wenige mittelständische Belegschaften ihre Mitwirkungs- und Mitbestimmungsrechte über Betriebsräte nutzen: Während in $93 \%$ der großen Betriebe die Mitarbeiter ihre Interessen durch Betriebsräte vertreten lassen, sind es im betriebsratsfähigen Mittelstand lediglich $10 \%$ der Betriebe (Sonderauswertung IAB-Betriebspanel 2004). Es stellt sich daher die Frage, warum so wenige Belegschaften in mittelständischen Unternehmen von der Wahl eines Betriebsrats Gebrauch machen.

Eine von mehreren Erklärungen hierfür könnte sein, dass andere Formen der Interessenvertretung in Unternehmen ohne Betriebsrat existieren, die eine Betriebsratsgründung nicht erforderlich machen. ${ }^{1}$ Erste Untersuchungen hierzu belegen die Existenz anderer Formen der Arbeitneh- mervertretung ${ }^{2}$ in Betrieben ohne Betriebsrat (IAB-Betriebspanel 2003; Martens 2003; Hauser-Ditz et al. 2006). Hierunter werden beispielsweise Runde Tische, Mitarbeiter-Ausschüsse, Mitarbeiter mit Moderatorenfunktion sowie Sprecher der Belegschaft gefasst.

Während die Verbreitung der unterschiedlichen Formen der Arbeitnehmervertretung im Mittelstand durch die genannten Erhebungen als relativ gut erfasst gelten kann, ist bis dato wenig über das Ausmaß der Beteiligung der jeweiligen Interessenvertretungen in mittelständischen Unternehmen bekannt. Im Folgenden sollen daher die Beteiligung und Mitsprache von Arbeitnehmern in mittelständischen Unternehmen mit unterschiedlichen Formen der Interessenvertretung einer Analyse unterzogen werden. Im Zentrum steht dabei die Frage, wie sich die Beteiligung der Arbeitnehmer in den verschiedenen Konstellationen von individuellen oder kollektiven Formen der Interessenvertretung im Mittelstand gestaltet und wie diese sich voneinander unterscheiden. Als empirische Grundlage dienen eine repräsentative Unternehmensbefragung sowie Fallstudien aus den Jahren 2005/2006. ${ }^{3}$

Der Beitrag ist wie folgt gegliedert: In Abschnitt 2 wird die Datenbasis kurz dargestellt. Abschnitt 3 geht auf die Verbreitung der verschiedenen Formen der Interessenvertretung in mittelständischen Unternehmen ein, bevor in Abschnitt 4 Formen und Ausmaß der Arbeitnehmerpartizipation in mittelständischen Unternehmen in Abhängigkeit von der jeweiligen Form der Interessenvertretung analysiert werden. Abschnitt 6 fasst die Befunde zusammen.

\footnotetext{
1 Neben der Existenz anderer Beteiligungsmöglichkeiten sind weitere Erklärungen für die geringe Zahl an Betriebsratsgründungen in mittelständischen Unternehmen denkbar wie bspw. Verhinderungsstrategien der Eigentümer oder Unwissenheit der Belegschaften über ihre Rechte.

2 Weitere Begriffe hierfür sind: interner Betriebsrat (Hilbert/Sperling 1993, S. 185ff.), betriebsspezifische Form der Mitarbeitervertretung (IAB-Betriebspanel 2003), informelle Vertretungsstrukturen (Martens 2003, S. 41) andere Vertretungsorgane (Hauser-Ditz et al. 2006, S. 500) oder andere Formen der Arbeitnehmervertretung (Schlömer et al. 2007).

3 Die Befragung sowie die Fallstudien wurden durch die Hans-Böckler-Stiftung gefördert und in der Schriftenreihe der Stiftung veröffentlicht: Schlömer et al. 2007.
}

Nadine Schlömer, Diplom-Kauffrau, wissenschaftliche Mitarbeiterin am Institut für Mittelstandsforschung Bonn. Arbeitsschwerpunkte: Personalpolitik in KMU sowie Unternehmensnachfolge

e-mail: schloemer@ifm-bonn.org Rosemarie Kay, Dr. rer. pol., Projektkoordinatorin am Institut für Mittelstandsforschung Bonn. Arbeitsschwerpunkte: Personalpolitik in KMU, Unternehmensgründung sowie Frauen als Gründerinnen und Unternehmerinnen

e-mail:kay@ifm-bonn.org

Wolfgang Rudolph, Soziologe M. A., wissenschaftlicher Mitarbeiter im Büro für Sozialforschung Kassel. Arbeitsschwerpunkte: Mitbestimmungsforschung, soziale Beziehungen in $\mathrm{KMU}$. e-mail: rudolph@bfs-kassel.de Wolfram Wassermann, Dr. rer. pol., wissenschaftlicher Mitarbeiter im Büro für Sozialforschung Kassel. Arbeitsschwerpunkte: Entwicklung des Betriebsrätewesens, gewerkschaftliche Betriebspolitik, Mittelstandsfragen.

e-mail: wassermann@bfs-kassel.de 


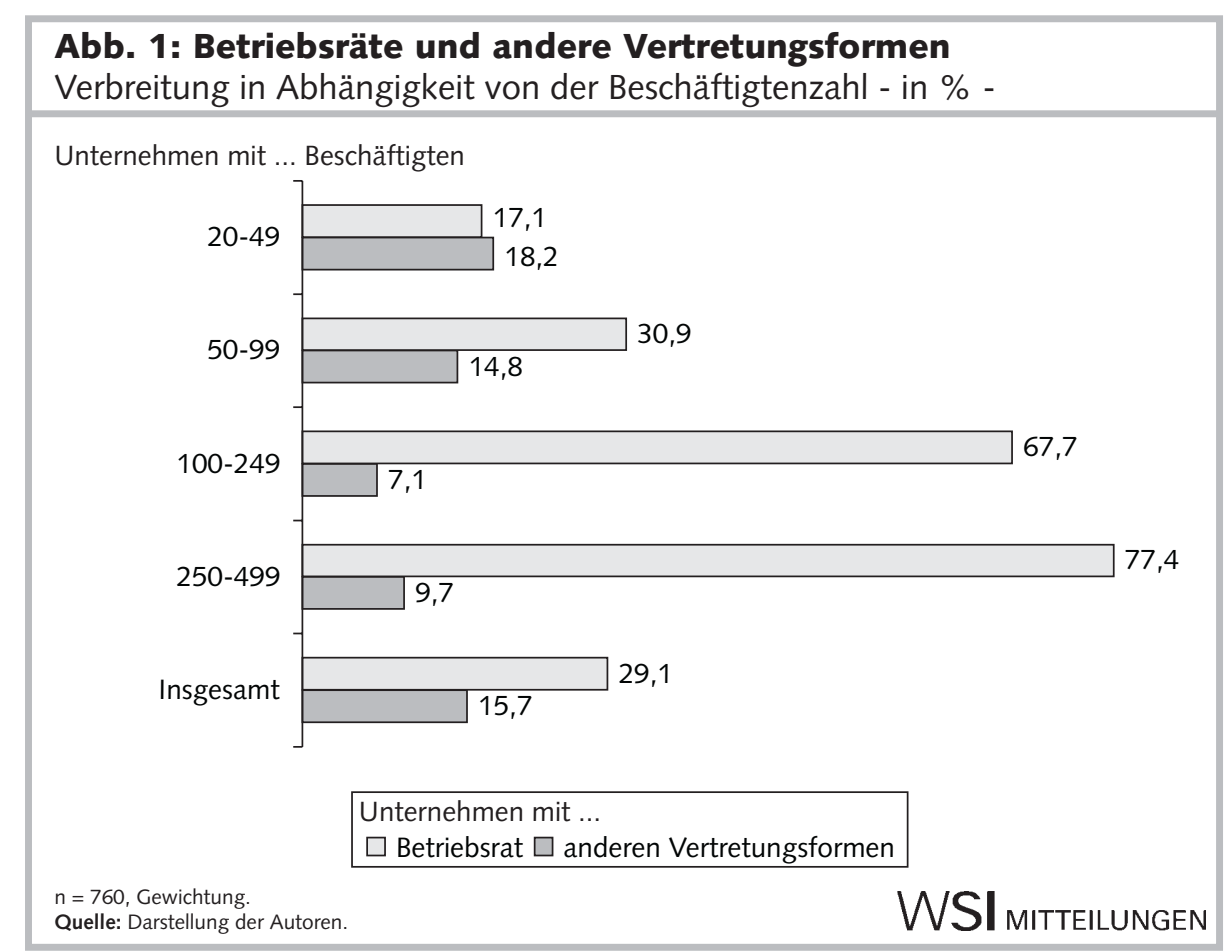

\section{Die Datenbasis}

Die empirische Untersuchung der Beteiligung und Mitsprache von Arbeitnehmern in mittelständischen Unternehmen erfolgt auf Basis repräsentativer Befragungen sowie betrieblicher Fallstudien. Befragt wurden sowohl Geschäftsführer als auch Arbeitnehmervertreter (Betriebsräte sowie Vertreter anderer Formen der Arbeitnehmervertretung) zur Praxis der betrieblichen Mitbestimmung und Partizipation. Für die folgenden deskriptiven Analysen werden ausschließlich Daten der Geschäftsführer-Befragung des Instituts für Mittelstandsforschung (IfM) Bonn verwendet, an der sich 809 mittelständische Geschäftsführer aus Unternehmen aller Branchen mit 20 bis 499 Beschäftigten beteiligt haben. Am stärksten vertreten sind Unternehmen, die überwiegend Dienstleistungen für andere Unternehmen erbringen, gefolgt von Unternehmen des Verarbeitenden Gewerbes, Bergbau und Energie. Knapp $17 \%$ der befragten Unternehmen sind der Kategorie große mittelständische Unternehmen (250 bis 499 Beschäftigte) zuzuordnen. Alle anderen Größenklassen (20 bis 49,50 bis 99 sowie 100 bis $249 \mathrm{Be}$ schäftigte) sind mit mindestens knapp $24 \%$ im Untersuchungssample vertreten. Etwa ein Fünftel der befragten Unterneh- von Arbeitnehmern, sei es in Form eines Betriebsrats oder einer anderen unternehmensspezifischen Form der Arbeitnehmervertretung (wie beispielsweise Runde Tische, Mitarbeiter-Ausschüsse, Sprecher der Belegschaft). In mehr als drei Fünfteln dieser Unternehmen wurde ein Betriebsrat gewählt. Andere Vertretungsformen wurden in knapp einem Drittel dieser Unternehmen eingerichtet. Eine Minderheit von knapp $5 \%$ hat sowohl einen Betriebsrat als auch eine andere Vertretungsform installiert. Bezogen auf alle Unternehmen der Größenklasse 20 bis 499 Beschäftigte ergibt sich folgende Verteilung (Abbildung 1): $29 \%$ der Unternehmen haben einen Betriebsrat, wohingegen knapp $16 \%$ der Unternehmen dieser Größenklasse über eine andere Form der Arbeitnehmervertretung verfügen. Andere Vertretungsformen sind demnach etwa halb so häufig in mittelständischen Unternehmen dieser Größenklasse anzutreffen wie Betriebsräte.

Die Verbreitung der verschiedenen Formen der kollektiven Interessenvertretung hängt u.a. von der Unternehmensgröße ab: So verfügen in der Größenklasse 20 bis 49 Beschäftigte etwa $18 \%$ der Unternehmen über eine andere Vertretungsform, während der Anteil der Unternehmen mit Betriebsrat in dieser Größenklasse bei $17 \%$ liegt. Anders stellt sich die Lage in der Größenklasse 250 bis 499 Beschäftigte dar: Hier ist in knapp $10 \%$ der mittelständischen Unternehmen eine andere Vertretungsform anzutreffen, während es in etwa drei Vierteln dieser Unternehmen einen Betriebsrat gibt.

Andererseits sind auch individuelle Formen der Interessenvertretung möglich. Das heißt, jeder Mitarbeiter vertritt seine Rechte selbst gegenüber der Geschäftsführung. Davon abzugrenzen ist die direkte Arbeitnehmer-Partizipation, die sich vornehmlich auf die Beteiligung der Arbeitnehmer an Entscheidungsprozessen innerhalb des operativen Geschäfts bezieht. Typische Bespiele für die direkte Partizipation sind Gruppenarbeit, Qualitätszirkel und Projektgruppen (Dörre 2001). In wie vielen der 57,2 \% mittelständischer Unternehmen, in denen keine kollektive Form der Interessenvertretung existiert, stattdessen individuelle Formen praktiziert werden, kann auf Basis des zugrundliegenden Datensatzes nicht ermittelt werden. 


\section{Formen und Ausmaß der Arbeitnehmerbeteiligung}

\subsection{UNTERNEHMEN MIT BETRIEBSRAT}

Grundlage für die Zusammenarbeit zwischen Betriebsrat und Geschäftsführung ist das Betriebsverfassungsgesetz (BetrVG). Neben den Rechten des Betriebsrats regelt dieses ferner die Zusammenarbeit zwischen den beiden Betriebsparteien. Nach $\$ 2$ BetrVG arbeiten Betriebsrat und Arbeitgeber vertrauensvoll zum Wohl der Arbeitnehmer und des Betriebs zusammen. Gleichwohl können die Geschäftsführer mittelständischer Unternehmen eine $\mathrm{Zu}$ sammenarbeit mit dem Betriebsrat auch vermeiden, wie Kotthoff/Reindl (1990) in ihren Fallstudien am Beispiel des, ,ignorierten Betriebsrats“ sowie des ,isolierten Betriebsrats" gezeigt haben. D.h. die Geschäftsführung hat grundsätzlich verschiedene Möglichkeiten, den Betriebsrat in Entscheidungsprozesse einzubeziehen: Erstens kann sie versuchen, einen Ausgleich der Interessen anzustreben, indem sie sich auf Kompromisse mit dem Betriebsrat verständigt. Eine weitere Möglichkeit ist die aktive Beteiligung, indem sie den Betriebsrat auch in Management-Entscheidungen von vornherein einbezieht. Drittens kann sie den Betriebsrat ignorieren und ihn bei Entscheidungsprozessen einfach übergehen - im Sinne des Ausschlusses. Viertens ist schließlich auch denkbar, dass Geschäftsführer versuchen, Entscheidungen gegen den Willen des Betriebsrats durchzusetzen (Schlömer et al. 2007).

Die im BetrVG verankerte kooperative Zusammenarbeit zwischen den beiden Betriebsparteien zeigt sich in der Mehrzahl der von uns untersuchten Unternehmen. Gut $90 \%$ der Geschäftsführer von Unternehmen mit Betriebsrat gaben an, dass sie bei organisatorischen Veränderungen den „Kompromiss“ oder die ,aktive Beteiligung" im Umgang mit dem Betriebsrat anstreben. Die Strategien „Ausschluss“ oder „Konfrontation“ haben eine eher randständige Bedeutung: So werden die Betriebsräte nur in 6,8 \% der betrachteten Unternehmen nicht in die Entscheidungen im Zusammenhang mit betrieblichen Veränderungsprozessen einbezogen, während die Strategie der Konfrontation nur in knapp $2,6 \%$ der Un-

Abb. 2: Beteiligung von Betriebsräten bei betrieblichen Veränderungen - in \% -

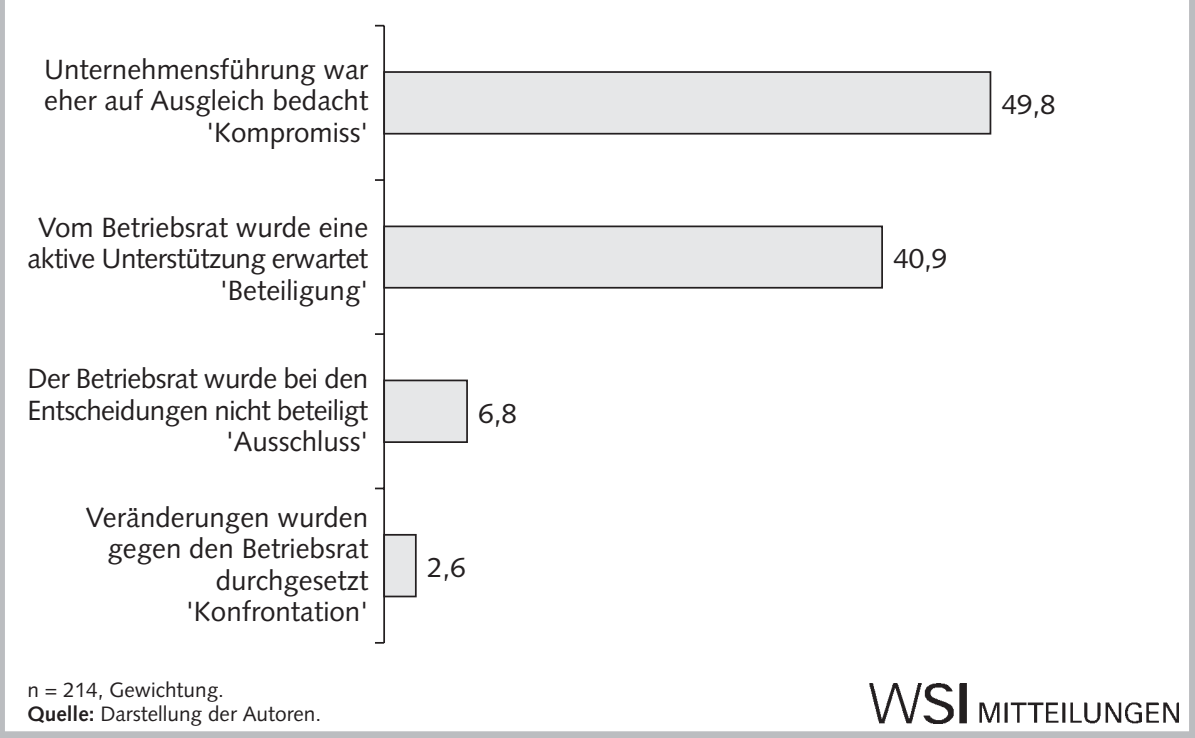

ternehmen mit Betriebsrat gewählt wird (Abbildung 2).

Gleichzeitig zeigt die schriftliche Unternehmerbefragung, dass die Geschäftsführer ihr Verhältnis zum Betriebsrat mit einem Mittelwert von 3,8 als eher gut bezeichnen. ${ }^{4}$ Dieses eher gute Verhältnis zwischen Geschäftsführer und Betriebsrat ist aber meist nicht von Anfang an vorhanden, sondern in der Regel das Ergebnis eines Reifungsprozesses (Wassermann 1999). Gefragt nach ihrer Meinung zum Betriebsrat zum Zeitpunkt seiner Gründung und zum heutigen Zeitpunkt geben die Geschäftsführer in der Befragung an, dass sie gegenüber dem Betriebsrat eine eher neutrale Haltung gehabt hätten (Mittelwert $=3,1$ ), die sich im Laufe der Zeit aber verbessert habe (Mittelwert $=3,4) .{ }^{5}$ Eine ähnliche Entwicklung im Verhältnis der Geschäftsführer zum Betriebsrat zeigte sich auch in den Fallstudien: In der Regel hat das Verhältnis mit erheblichen Konflikten begonnen. Beide Seiten berichten von großen Eklats auf Betriebsversammlungen und von „Eiszeiten“, in denen monatelang nicht miteinander gesprochen wurde. Kraftproben und wechselseitige Erfahrungen mit dem Verhalten des anderen in Konfliktsituationen haben beide Seiten schließlich doch zusammen gebracht. Sie achten sich nun als Partner oder als Gegner und vertrauen darauf, dass die andere Seite auch in zugespitzten Krisensituationen letztlich verantwortungsvoll im Sinne des Betriebswohls handelt. Die gleichwohl zunächst verhaltene Haltung gegenüber dem
Betriebsrat könnte daher rühren, dass die Gründung von zwei Dritteln aller Betriebsräte im betrachteten Mittelstand von den Arbeitnehmern selbst - teilweise mit Unterstützung der Gewerkschaften - ausging. Lediglich in 22,4\% der Fälle war die Geschäftsführung an der Gründung eines Betriebsrats beteiligt. Zudem weisen die Umstände der Gründung auf mögliches Konfliktpotenzial hin: So werden Betriebsräte häufig im Zuge betrieblicher Umbruchsituationen (Eigentümerwechsel, Organisationsänderungen) und aufgrund des verstärkten Wunsches der Mitarbeiter nach mehr Beteiligung gegründet (Schlömer et al. 2007).

Das erst im Zeitverlauf entstandene mehrheitlich gute Verhältnis zwischen Betriebsrat und Geschäftsführung gibt jedoch noch keine Auskunft darüber, wie der Betriebsrat seine Mitwirkungs- und Mitbestimmungsrechte in Anspruch nimmt. Sowohl die schriftliche Befragung als auch die Fallstudien zeigen, dass die Betriebsräte nicht alle durch das Betriebsverfassungsgesetz zugesicherten Rechte nutzen. So werden in einem Drittel (32,3\%) aller Unternehmen mit 20 bis 499 Beschäftigten die Absprachen mit dem Betriebsrat mündlich

\footnotetext{
4 Die Antworten der Geschäftsführer wurden mittels einer 5 er Skala gemessen. Antwortausprägungen: $1=$ sehr schlecht und $5=$ sehr gut.

5 Die Antworten der Geschäftsführer wurden mittels einer 5er Skala gemessen. Antwortausprägungen: $1=$ sehr negativ und $5=$ sehr positiv .
} 
Tabelle 1: Andere regelmäßige Formen der kollektiven Interessenvertretung: Kreis der Vertretenen und Art der Gründung - in \% -

\begin{tabular}{lcrrrr|} 
& & \multicolumn{3}{c}{ Art der Gründung } & Gesamt \\
& & Gewählt & Ernannt & $\begin{array}{c}\text { Selbst- } \\
\text { ernannt }\end{array}$ \\
\hline Kreis der Vertretenen & Alle Beschäftigten & 43 & 14 & 13 & 70 \\
& Nicht alle Beschäftigten & 23 & 6 & 1 & 30 \\
\hline Gesamt $(n=79)$ & & 66 & 20 & 14 & 100 \\
\hline \multirow{2}{*}{ Quelle: IfM Bonn Befragung der Geschäftsführung. } & & & W/S I MITTEILUNGEN
\end{tabular}

getroffen. Formlose Absprachen sind vor allem dort üblich, wo im Laufe der Jahre ein enges Vertrauensverhältnis zwischen Inhaber und Betriebsratsvorsitzendem entstanden ist. Man einigt sich „per Handschlag“. Die Ausarbeitung komplizierter schriftlicher Vertragswerke meiden beide Seiten. Ein Betriebsratsvorsitzender betonte in den Fallstudien, entscheidend für den wenig formellen Verhandlungsstil sei für ihn die Tatsache, dass die Geschäftsführung bisher noch stets jede ihrer mündlichen Zusagen eingehalten habe.

Die schriftliche Befragung zeigt weitere Abweichungen vom Gesetz: So weisen $13 \%$ der mittelständischen Unternehmen mit Betriebsrat eine gerade Zahl an Betriebsratsmitgliedern auf, während das BetrVG nur eine ungerade Zahl an Betriebsratsmitgliedern vorsieht. Danach gefragt, ob es in den gemeinsamen Verhandlungen in den letzten drei Jahren zu einer einvernehmlichen Unterschreitung tariflicher Ansprüche gekommen sei, bejahen dieses 29,8 \% der Geschäftsführer. Abweichung von der gesetzlichen Vorschrift kann aber auch eine erweiterte Partizipation bedeuten. Etwa $42 \%$ der mittelständischen Geschäftsführer beteiligen nach eigenen Angaben ihren Betriebsrat „oft" bis ,immer“ über die mitbestimmungspflichtigen Bereiche hinaus an anderen betrieblichen Entscheidungen.

\subsection{UNTERNEHMEN MIT ANDEREN FORMEN DER ARBEITNEHMER- VERTRETUNG}

Bei der Bewertung der „alternativen“ "Vertretungsformen, die in der Regel anstatt eines Betriebsrats in den mittelständischen Unternehmen ins Leben gerufen wurden, besteht die Gefahr, diese vorschnell als „,betriebsratsähnliche" Organe zu kategorisieren. Im Rahmen der schriftlichen Befragung gaben etwa $51 \%$ der Unternehmen mit einer anderen Arbeitnehmervertretung an, dass diese nur eine ad hoc-Erscheinung sei. Das heißt, sie sind aus einem bestimmten Anlass (z. B. Konflikt) entstanden und hatten danach keinerlei Funktion mehr in den Unternehmen.

In den restlichen $49 \%$ der Unternehmen mit anderen Formen der Arbeitnehmervertretung stellen diese alternativen Formen jedoch eine feste Institution dar, die regelmäßig Aufgaben im Unternehmen übernimmt. Hierbei dominieren Gremien, in denen sowohl die Arbeitnehmer als auch die Geschäftsführung vertreten sind. So sind „regelmäßige Aussprachen mit Mitarbeitern" (91\%) und „Runde Tische“6 (59\%) die häufigsten Erscheinungsformen. Reine Arbeitnehmervertretungen, wie den Mitarbeiter-Ausschuss, den Belegschaftssprecher oder Mitarbeiter mit Moderatorenfunktion, gibt es in knapp der Hälfte (46\%) der mittelständischen Unternehmen. ${ }^{7}$ Das heißt, in der Mehrzahl der Fälle handelt es sich bei den anderen Vertretungsformen um Gremien, in denen sowohl die Arbeitnehmer als auch die Geschäftsführung vertreten sind. Ein Beispiel: In einem Bauunternehmen mit rund 60 Beschäftigten hat der Inhaber vor ein paar Jahren als Ersatz für den Betriebsrat eine „Polierrunde“ geschaffen. Die Runde aus Polieren sowie technischen und kaufmännischen Leitern tritt zwei Mal jährlich zusammen. Sie tagt jeweils zwei bis drei Stunden. Wichtige betriebliche Entscheidungen, einschließlich Entlassungen, Arbeitszeitveränderungen oder Veränderungen bei den Löhnen werden hier vorgeklärt. Wenn der Inhaber Entlassungen aus persönlichen Gründen für nötig hält, trägt er seine Gründe bisweilen vor und holt sich die Zustimmung der Poliere. Offene Aussprachen sind in der Polierrunde üblich, auch wenn die Letztentscheidung beim Inhaber verbleibt. Hier tritt ein Unterschied zu Unternehmen mit einem Betriebsrat deutlich zutage. Da Kündigungen mitbestimmungspflichtig sind, können Arbeitgeber in Unternehmen mit Betriebsrat - anders als hier in dem Fall- beispiel - die Letztentscheidung nicht selbst treffen.

Weitere Unterschiede zum Betriebsrat ergeben sich, weil die anderen Vertretungsformen nicht nach dem Betriebsverfassungsgesetz legitimiert sind und folglich keine gesetzlich verbrieften Rechte haben. Dies führt u.a. dazu, dass es weder Vorgaben für die Gründung noch den Kreis der Vertretenen dieser alternativen Arbeitnehmervertretungen gibt. Tabelle 1 gibt Auskunft, wie andere regelmäßige Formen der Arbeitnehmervertretung entstanden sind und welchen Kreis der Mitarbeiter sie vertreten. Es zeigt sich, dass regelmäßige andere Vertretungsformen, die wie ein Betriebsrat von allen bzw. von Teilen der Belegschaft gewählt werden und darüber hinaus auch alle Mitarbeiter des Unternehmens vertreten, mit $43 \%$ den größten Anteil unter den anderen regelmäßigen Vertretungsformen ausmachen.

Weitere Ähnlichkeiten zwischen den beiden Formen der kollektiven Interessenvertretung zeigen sich im Hinblick auf die Zusammenarbeit mit der Geschäftsführung. In beiden Fällen stellen kooperative Formen mit einem Anteil von über $90 \%$ die Hauptform der Zusammenarbeit dar. Anders als in Betrieben mit Betriebsrat dominiert in Unternehmen mit anderen Vertretungsformen die ,aktive Beteiligung“ als Umgangsform (54,4 \%). Der „Kompromiss" ist die zweitwichtigste Form der $\mathrm{Zu}$ sammenarbeit. Die Beteiligungsstrategien „Ausschluss“ oder „Konfrontation“ haben eine noch randständigere Bedeutung als bei den Unternehmen mit Betriebsrat: So werden die anderen Vertretungsformen nur in 5,9\% der Unternehmen mit regelmäßiger anderer Arbeitnehmervertretung nicht in Entscheidungen im Zusammenhang mit betrieblichen Veränderungsprozessen einbezogen. Die Strategie der „Kon-

\footnotetext{
6 Runde Tische sind Verfahren, bei denen Vertreter verschiedener Interessengruppen an einem Tisch zusammenkommen, um einen bestimmten Aufgabenkatalog zu bearbeiten und im Konsens zu lösen.

7 Durch die Filterführung im Fragebogen konnte sichergestellt werden, dass nur solche Vertretungsformen angegeben werden, die wirklich eine indirekte Form der Partizipation darstellen. So wurden die Befragten zunächst gefragt, ob es überhaupt eine andere Vertretungsform gibt, welche die Interessen der Arbeitnehmer gegenüber der Geschäftsführung vertritt. Erst wenn diese Frage mit ja beantwortet worden ist, wurden den Befragten Auswahlmöglichkeiten vorgegeben.
} 
frontation" wird nur in 1,5\% dieser Unternehmen gewählt.

Deutlich besser als Geschäftsführer in Unternehmen mit Betriebsrat bewerten Geschäftsführer von Unternehmen mit anderen Vertretungsformen ihr Verhältnis zur eigenen Arbeitnehmervertretung. Im Durchschnitt bezeichnen sie das Verhältnis als gut bis sehr gut (Mittelwert $=4,4$ ), was vor dem Hintergrund, dass fast $80 \%$ aller anderen Vertretungsformen auf Initiative der Geschäftsführung entstehen, nicht verwundert. ${ }^{8}$ So überrascht es auch nicht, dass das Verhältnis - anders als bei Betriebsratsstrukturen - von Beginn an gut war, sich gleichwohl aber noch verbessert hat. ${ }^{9}$ Dass die Beziehung von alternativer kollektiver Vertretung und Geschäftsführung von Beginn an als positiv eingeschätzt wird, hat allerdings nicht nur mit der angesprochenen Initiative der Geschäftsführung zu tun, sondern auch mit dem Entstehungskontext alternativer Arbeitnehmerbeteiligungen: Während Betriebsratsgründungen im Mittelstand am ehesten erfolgen, wenn Umbruchsituationen drohen bzw. wenn die Mitarbeiter verunsichert sind oder sich mehr Beteiligung wünschen, sollen mit der Gründung alternativer kollektiver Formen Mitarbeiter motiviert bzw. das Unternehmen gestärkt werden (Schlömer et al. 2007). Ein vertrauensvolles Miteinander ist folglich die Basis, was sich in der Art und Weise, wie Absprachen getroffen werden, widerspiegelt: Deutlich häufiger als beim Betriebsrat werden Absprachen mit dem Geschäftsführer nur mündlich vereinbart (84,9\%).

In Konfliktsituationen wiederum bleibt den alternativen kollektiven Interessenvertretungen in Ermangelung einer Rechtsgrundlage nur der Weg des Kompromisses.

Lassen sich Arbeitgeber jedoch nicht auf Kompromisse ein, wird die Gründung eines Betriebsrats für die Belegschaften zu einer Option. Eine Betriebsratsgründung wird für Vertreter von anderen regelmäßigen Vertretungsformen zudem denkbar, wenn ihre bisherigen Rechte nicht mehr ausreichen, wie z. B. im Falle von anstehenden Betriebsschließungen oder beim Abschluss von Betriebsvereinbarungen (Schlömer et al. 2007). Entsprechend zeigt die Erhebung von Hauser-Ditz et al. (2007), dass $23 \%$ der Betriebe mit Betriebsrat zuvor über eine andere alternative kollektive Interessenvertretung verfügten.

\section{Abb. 3: Beteiligung von anderen regelmäßigen Vertretungsformen}

bei betrieblichen Veränderungen - in \% -

Unternehmensführung war
eher auf Ausgleich bedacht
'Kompromiss'

\subsection{UNTERNEHMEN OHNE FORMALISIERTE ARBEITNEHMER- VERTRETUNGEN}

Etwa 57 \% der mittelständischen Unternehmen mit 20 bis 499 Beschäftigten verfügen - wie in Abschnitt 3 angesprochen weder über einen Betriebsrat noch über ein anderes identifizierbares Vertretungsorgan. Dies ist jedoch nicht zwangsläufig mit einem Verzicht der Beschäftigten, ihre Interessen zu vertreten, gleichzusetzen. Direkte, d.h. individuelle Formen der Arbeitnehmervertretung, die eine mögliche weitere Form der Arbeitnehmerbeteiligung darstellen, sind jedoch im Rahmen schriftlicher Unternehmensbefragungen nur schwer erfassbar. Deshalb können zur Klärung der Beteiligungssituation in Unternehmen ohne jegliche Form von Arbeitnehmervertretung vornehmlich die Fallstudien Hinweise geben. Und tatsächlich zeigt sich hier, dass gerade in kleinen Betrieben die direkte Arbeitnehmerbeteiligung selbstverständlicher Bestandteil des täglichen Arbeitslebens sein kann. Ein Beispiel: In einem kleinen Metallbetrieb, der 25 Arbeitnehmer beschäftigt, hat der Gründer und Inhaber der Firma lediglich einen Prokuristen an seiner Seite. Soziale Fragen oder Konflikte werden hier zwischen den Mitarbeitern und dem Inhaber bzw. dem Prokuristen direkt besprochen. Dabei geht es meist um Wünsche nach Lohnerhöhung oder Hilfe in schwierigen familiären Situationen.

Ähnlich gestaltet sich die Situation in einem ländlich gelegenen Metallunterneh- men mit fast 180 Beschäftigten. Es existiert kein Betriebsrat und der Inhaber glaubt, dies sei der Erfolg seiner, wie er es ausdrückt, „partnerschaftlichen Personalarbeit". Der Inhaber selbst hält durch regelmäßige Betriebsrundgänge den Kontakt zur Belegschaft. Er hat alle seine Abteilungsleiter angewiesen, dies in ihren Bereichen ebenso zu tun. Er nennt diese Methode „Management by walking around“. ${ }^{10}$ Aufkeimende Spannungen und Konflikte sollen so bereits im Frühstadium erkannt und entschärft werden. Darüber hinaus bietet die Firma ihren Beschäftigten ein breites Spektrum von sozialen Angeboten, Praktiken und Einrichtungen: Kinderkrippe und Ferienprogramme für Kinder von Beschäftigten, Weiterbildungsangebote etwa zur Bewältigung von Alltagskonflikten in Betrieb und Familie. Hinzu kommen freiwillige Arbeitskreise, in denen die Be-

8 Die Antworten der Geschäftsführer wurden mittels einer 5 er Skala gemessen. Antwortausprägungen: $1=$ sehr schlecht und $5=$ sehr gut.

9 Gefragt nach ihrer Meinung zur Arbeitnehmervertretung zum Zeitpunkt ihrer Gründung und zum heutigen Zeitpunkt geben die Geschäftsführer in der Befragung an, dass sie gegenüber der alternativen Arbeitnehmervertretung eine neutrale bis eher positive Haltung hatten (Mittelwert $=3,7$ ), die sich im Laufe der Zeit noch verbessert habe (Mittelwert =3,9). Die Antwort der Geschäftsführer wurde mittels einer $5 \mathrm{er}$ Skala gemessen. Antwortausprägungen: $1=$ sehr negativ und $5=$ sehr positiv.

10 "MBWA ${ }^{\prime}$ ist der Name einer aus den USA stammenden Managementlehre (Peters/Waterman 1982) 
schäftigten sich für betriebliche und soziale Belange einsetzen können. In einem solchen Arbeitskreis wurde aber auch eine neue Gleitzeitregelung erarbeitet, die nun im Betrieb angewandt wird.

Die beiden dargestellten Formen von individueller Interessenvertretung stellen sicherlich Positivbeispiele dar, weil die Mitarbeiter Mitwirkungs- und Mitbestimmungsrechte wahrnehmen können, die ansonsten ein Betriebsrat ausübt. Allerdings: Es geht stets nur um einen Teilbereich der Mitwirkungs- und Mitbestimmungsrechte, die dem Betriebsrat nach dem BetrVG zustehen. Und außerdem: Solche direkten Beteiligungsmöglichkeiten werden sich nicht in jedem Unternehmen ohne kollektive Formen der Interessenvertretung finden. Im Hinblick auf die Regelung von Konflikten wird bei den individuellen Formen der Arbeitnehmervertretung - wie auch bei den alternativen kollektiven Interessenvertretungen - der Kompromiss mangels rechtlicher Möglichkeiten die gängige Vorgehensweise sein. Auch hier wird die Betriebsratsgründung bei fehlender Kompromissbereitschaft der Geschäftsführung bzw. fehlenden rechtlichen Gestaltungsmöglichkeiten zu einer Option für die Belegschaften.

\section{Zusammenfassung}

Mittelständische Belegschaften nutzen selten den Betriebsrat als Form der kollektiven Interessenvertretung. Um dieses Phänomen besser $\mathrm{zu}$ verstehen, haben wir Verbreitung und Form der Arbeitnehmer- beteiligung in unterschiedlichen Beteiligungskonstellationen in mittelständischen Unternehmen näher analysiert. Die Ausgangsthese war, dass es andere Formen von Interessenvertretung gibt, die einen Betriebsrat obsolet machen.

In einem knappen Drittel der mittelständischen Unternehmen mit 20 bis 499 Beschäftigten findet Arbeitnehmerbeteiligung über Betriebsräte statt. Wie im BetrVG vorgegeben, gestaltet sich die $\mathrm{Zu}$ sammenarbeit zwischen Geschäftsführung und gesetzlicher Arbeitnehmervertretung in der Mehrzahl der mittelständischen Unternehmen kooperativ. Das Ausmaß der Beteiligung unterscheidet sich trotz der Vorgaben des BetrVG jedoch von Unternehmen zu Unternehmen, weil die jeweiligen Betriebsräte ihre gesetzlich zugesicherten Rechte in unterschiedlichem Maße in Anspruch nehmen.

In knapp jedem sechsten mittelständischen Unternehmen wurden andere Formen der indirekten Arbeitnehmervertretung installiert. Jedoch lediglich jede zweite dieser alternativen Formen der Arbeitnehmervertretung ist von Dauer. Die Zusammenarbeit zwischen regelmäßiger alternativer Arbeitnehmervertretung und Geschäftsführung ist wie beim Betriebsrat kooperativer Natur, wobei jedoch diese alternative Arbeitnehmervertretung von der Geschäftsführung stärker aktiv eingebunden wird. Dies kann darin begründet liegen, dass durch die Mitgliedschaft der Geschäftsführung in der Mehrheit der anderen Formen der kollektiven Interessenvertretungen die oppositionelle Stellung beider Betriebsparteien zueinander weniger stark ausgeprägt ist als bei Vorhandensein eines Betriebsrats.
Mehr als die Hälfte der mittelständischen Unternehmen verfügt über keine formalisierte Arbeitnehmervertretung. In einigen dieser Unternehmen haben sich individuelle Formen der Interessenvertretung etabliert, die den Mitarbeitern ebenfalls Mitsprache in einigen sozialen und personalen Angelegenheiten ermöglichen - die aber nur einen Teilaspekt der Mitbestimmungsbereiche eines Betriebsrats abdecken.

Die Beteiligungssituation in Unternehmen mit regelmäßigen kollektiven sowie mit individuellen Formen der Interessenvertretung weist unterschiedlich starke Ähnlichkeiten zu den Strukturen mit Betriebsrat auf. Am meisten ähneln andere regelmäßige Vertretungsformen Betriebsratsgremien, wenn sie von allen Mitarbeitern gewählt werden und diese auch vor der Geschäftsführung vertreten.

Wie eingangs vermutet, scheint der mögliche Rückgriff auf andere Interessenvertretungsformen - sei es über alternative kollektive oder individuelle - dazu zu führen, dass eine Betriebsratsgründung den mittelständischen Belegschaften nicht mehr primär erforderlich erscheint. Typisch ist aber auch, dass diese Einschätzung im Konfliktfall umschlägt: Wenn eine Belegschaft mit alternativer kollektiver bzw. individueller Interessenvertretung feststellt, dass ihre bisherigen Rechte durch betriebliche Umbrüche gefährdet sind oder Probleme mit den bisherigen Mitteln nicht mehr gelöst werden können, dann verstärkt sich das Interesse, einen Betriebsrat im Sinne des BetrVG zu konstituieren. 
Dörre, K. (2001): Partizipation im Arbeitsprozess: Alternative oder Ergänzung zur Mitbestimmung, in: Industrielle Beziehungen 4, S. 379-407 Hauser-Ditz, A./Hertwig, M./Pries, L. (2006): Betriebsräte und andere Vertretungsorgane im Vergleich - Strukturen, Arbeitsweisen und Beteiligungsmöglichkeiten, in: WSI Mitteilungen 9, S. 500-506

Hauser-Ditz, A./Hertwig, M./Pries, L. (2007): Ergebnisse der BISS-Befragung 2005 - unveröffentlichter Projektbericht

Hilbert, J./Sperling, H. J. (1993): Die kleine Fabrik: Beschäftigung,

Technik und Arbeitsbeziehungen, München und Mering

IAB-Betriebspanel (2003): Fragebogen 2003, S. 21. http://doku.iab.de/ $\mathrm{fdz} / \mathrm{iabb} / \mathrm{fb} \_2003 . p d f$

IAB-Betriebspanel (2004): Sonderauswertung Betriebe mit Betriebsrat nach Betriebsgröße 2004. IAB-Betriebspanel 12. Welle West, 9. Welle Ost

Kotthoff, H./Reindl, J. (1990): Die soziale Welt kleiner Betriebe: Wirtschaften, Arbeiten und Leben im mittelständischen Industriebetrieb, Göttingen
Martens, H. (2003): Primäre Arbeitspolitik und Interessenvertretung in der New Economy, Beiträge aus der Forschung 137, Sozialforschungsstelle Dortmund

Minssen, H. (1999): Direkte Partizipation contra Mitbestimmung?

Herausforderung durch diskursive Koordinierung, in: Müller-Jentsch, W. (Hrsg.): Konfliktpartnerschaft, München und Mering, S. 129-156

Niedenhoff, H.-U. (2005): Mitbestimmung im europäischen Vergleich, in: IW-Trends 2, S. 3-17

Peters, T. J./Waterman, R. H. (1982): In Search of Excellence: lessons from America's best-run companies, New York Schlömer, N./Kay, R./Backes-Gellner, U./Rudolph, W./Wassermann, W. (2007): Mittelstand und Mitbestimmung - Unternehmensführung, Mitbestimmung und Beteiligung in mittelständischen Unternehmen, Münster Wassermann, W. (1999): Kampf den mitbestimmungsfreien Zonen? Überlegungen zu einer den Bedingungen in Kleinbetrieben angemessenen Weiterentwicklung der Betriebsverfassung, in: WSI Mitteilungen 11, S. $770-782$ 